\title{
Bare-Tether Sheath and Current: Comparison of Asymptotic Theory and Kinetic Simulations in Stationary Plasma
}

\author{
Juan R. Sanmartín, Eric Choinière, Brian E. Gilchrist, Jean-Benoit Ferry, and Manuel Martínez-Sánchez
}

\begin{abstract}
Analytical expressions for current to a cylindrical Langmuir probe at rest in unmagnetized plasma are compared with results from both steady-state Vlasov and particle-in-cell simulations. Probe bias potentials that are much greater than plasma temperature (assumed equal for ions and electrons), as of interest for bare conductive tethers, are considered. At a very high bias, both the electric potential and the attracted-species density exhibit complex radial profiles; in particular, the density exhibits a minimum well within the plasma sheath and a maximum closer to the probe. Excellent agreement is found between analytical and numerical results for values of the probe radius $R$ close to the maximum radius $R_{\max }$ for orbital-motion-limited (OML) collection at a particular bias in the following number of profile features: the values and positions of density minimum and maximum, position of sheath boundary, and value of a radius characterizing the no-space-charge behavior of a potential near the high-bias probe. Good agreement between the theory and simulations is also found for parametric laws jointly covering the following three characteristic $R$ ranges: sheath radius versus probe radius and bias for $R \ll R_{\max }$; density minimum versus probe bias for $R \cong R_{\max }$; and (weakly bias-dependent) current drop below the OML value versus the probe radius for $R>R_{\text {max }}$.
\end{abstract}

Index Terms-Asymptotic theory, bare electrodynamic tether, high-voltage sheath, Langmuir probe current, particle-in-cell (PIC) simulations, steady-state Vlasov simulations.

\section{INTRODUCTION}

$\mathbf{T}$ HE BARE-TETHER concept, i.e., leaving a conductive space tether bare of insulation to freely collect charge from the ambient plasma [1], has spurred new issues in the old theory of cylindrical Langmuir probes, which have long been used in space. At a negative bias $\Phi_{p}$, with $-e \Phi_{p} \sim k T_{e}$, the retarding range of the probe characteristic has served to determine the electron temperature $T_{e}$; ion collection, which is dominant at a more negative bias, has been used to determine the plasma density $n_{\infty}$ [2]. In the past, studies of the effects of relative plasma velocity $U_{0}$ were limited to moderate voltages [3]; when the probe was nearly aligned with the spacecraft velocity, the ion current showed a peak that can serve to find the ion temperature $T_{i}$ [4], [5]. Typically, however, a bare tether operates as a giant Langmuir probe at a positive bias, with $e \Phi_{p}$ being very much greater than both the thermal energy of particles and the ion ram energy. The probe sheath is then much larger than both the probe radius $R$ and the Debye length $\lambda_{D}$, whereas the latter two quantities may have similar values.

In the absence of effects from the geomagnetic field $B_{0}$ and from the velocity $U_{0}$, both the potential $\Phi(r)$ and electron density $n_{e}(r)$ have been theoretically predicted to exhibit spatially complex structures [6], [7]. The faraway potential behaves as $\Phi \sim 1 / r$, this being just a result from the angle subtended by the probe decreasing as inverse distance $1 / r$ and as $\Phi \sim \ln r$ inside some neighborhood around the probe, where the high bias makes space charge effects negligible. In between, however, the product $\Phi r^{2}$ would go through a relative minimum at a certain point 0 lying outside the sheath, with $\Phi_{0} r_{0}^{2}>\Phi_{p} R^{2}$ in the case where the probe is collecting orbital-motion-limited (OML) currents and through a maximum at some point $m$ well inside the sheath (Fig. 1). The density itself would show a minimum $n_{\min }$ at point $m$ and a maximum near point $Q$, where $\Phi_{Q} r_{Q}^{2}=\Phi_{0} r_{0}^{2}$, close to the probe before settling at a value of $1 / 2 n_{\infty}$ at its surface. For $R$ that is greater than some maximum radius $R_{\max }$, point 0 lies below the diagonal in Fig. 1, the density at the probe drops below $1 / 2 n_{\infty}$, and the current drops below the OML value. This paper proposes to validate both the analytical and numerical approaches by comparing the results on sheath profile features and on parametric laws, such as sheath radius and current beyond the OML regime.

Except for the geometry-specific Laplace-type potential near the probe, the results for arbitrary convex cross sections (with some definite "equivalent radius" $R_{\text {eq }}$ playing the role of the radius of a circular cross section) are quite similar [8]. The 2-D OML law for electron collection is very robust. It does not require azimuthal symmetry; it is independent of the unperturbed ion distribution and (at high bias) is also independent of the electron distribution if isotropic, as with the highly subsonic 


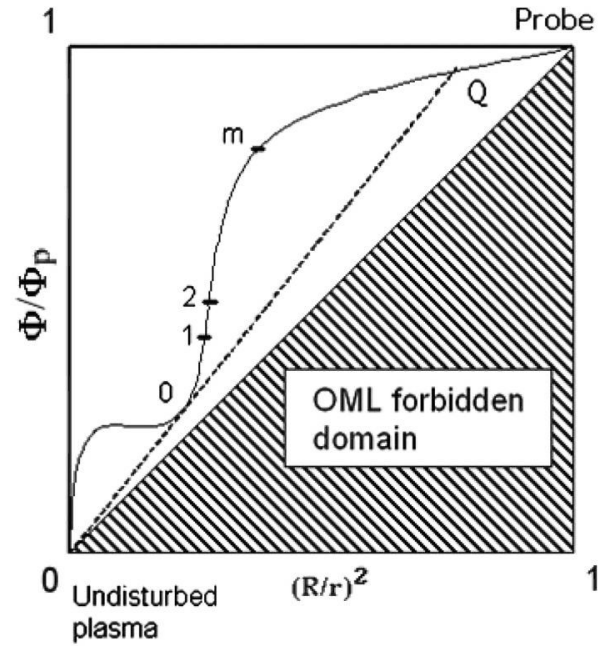

Fig. 1. Schematics of potential profile for a cylindrical probe in quiescent unmagnetized plasma in the case of OML current collection (point 0 above the diagonal).

relative electron flow at low Earth orbit (LEO). Moreover, the probe radius can be made much smaller than the electron thermal gyroradius. Ignoring both $U_{0}$ and $B_{0}$ might thus possibly be a valid approach in predicting electron current collection in LEO. Preliminary laboratory tests at the Marshall Space Flight Center have been followed by more careful tests [9]-[12]; however, the full laboratory simulation of all conditions in space is difficult. Both particle-in-cell (PIC) [13]-[15] and steady-state Vlasov [16], [17] numerical analyses have been carried out, with results being (as of yet) inconclusive about a collected-current law for magnetized $\left(B_{0} \neq 0\right)$ flowing $\left(U_{0} \neq 0\right)$ plasma.

A recent steady-state Vlasov analysis of highly biased ionattracting probes has provided detailed sheath profile and current collection results for both stationary and flowing plasma, with $B_{0}=0$ [18]. In this paper, we present a thorough comparison of theoretical results from [6] and [7] with numerical results for the stationary $\left(U_{0}=0\right)$ case from [18] and [14]. Note that the LEO $U_{0} \neq 0$ case is fundamentally different for negative and positive biases, owing to the mesothermal character of the relative plasma flow [19]. Note also that the OML collection appears applicable in unmagnetized plasma as long as the attracted species has an isotropic distribution function [20].

\section{Kinetic Modeling of High-Voltage PRobe SHEATHS}

Theoretical results will first be compared with some recently published numerical results pertaining to high-voltage sheaths [18], which were obtained using a steady-state self-consistent kinetic model implemented as the kinetic plasma solvers (KiPS) [17]. The simulation results shown in [18] include the sheath profile and collected ion current and pertain to both stationary and flowing plasma with Mach numbers from 0 to 10. For the present purposes, we will only use the stationary (nonflowing) kinetic simulation results based on KiPS-1-D simulations, which span the bias ratios $e \Phi_{p} / k T_{e}$ ranging from -10 to -5120 , whereas results with earlier similar models [21] had been provided over a limited range of potential bias ratio (down to -25$)$.

KiPS-1-D and KiPS-2-D are steady-state self-consistent kinetic solvers that are capable of simulating, respectively, stationary and flowing collisionless unmagnetized plasma in a vast region surrounding a round conductive cylinder of an arbitrary radius (in the case of KiPS-1-D) or any 2-D conductive object (in the case of KiPS-2-D). The KiPS codes numerically solve, in a self-consistent manner, the Poisson and Vlasov equations in the steady state. The Poisson solver was implemented using the finite-element method. In 1-D, the Vlasov solver uses conservation of energy and angular momentum to infer velocity-distribution functions within the computational zone. In 2-D, the Vlasov solver is based on the inside-out approach of tracking particle trajectories backward in time up to their origin on one of the computational-space boundaries.

A key component of the solvers is their iterative approach to reaching a self-consistent solution based on successive linearizations of the nonlinear Poisson-Vlasov operator [17]. Numerical instabilities resulting from the large grid sizes required to model the large sheaths resulting from high voltages are handled using a Tikhonov-regularized Newton iterative process [17]. The sharp features encountered in the net space charge near the sheath edge in high-voltage sheaths are accurately resolved in both KiPS-1-D and KiPS-2-D using an adaptive meshing strategy.

Theoretical results will also be compared with some results from [14], which were obtained using a PIC method, which groups particles into superparticles having parameters defined as the sum of the corresponding parameters of all particles in the cell. From the superparticle positions, the plasma density is extrapolated to each node of the grid and then used in the Poisson equation to calculate the potential. The electric field and resulting acceleration are then inferred for each superparticle, allowing one to update its velocity and position. The PIC method could not be used, however, in the proximity of a highly biased tether due to the high velocity of superparticles with respect to the scale of the grid. Instead, the motion of electrons inside a small domain around the tether was calculated analytically, using local conservation of energy and angular momentum under a fairly valid assumption of a purely Laplacian field.

With regard to boundary conditions, quasi-neutrality was imposed at the boundary of the simulation domain, rewriting separately the densities of incoming and outgoing electrons and ions $n_{e}^{\text {in }}+n_{e}^{\text {out }}=n_{i}^{\text {in }}+n_{i}^{\text {out }}$. In the case of no flow and no magnetic field, incoming densities could be obtained by integrating Maxwell-Boltzmann distributions, whereas outgoing densities were computed from the simulation itself. Combining both types of expressions allowed for the calculation of the potential at the boundary, which was then used to evaluate the velocity of new particles to be injected.

\section{Comparison of Sheath Profile Features}

To readily refer to theoretical results from [6], we consider the case of an electron-attracting probe, which is now entirely symmetric to the ion-attracting case considered in [18]. The 


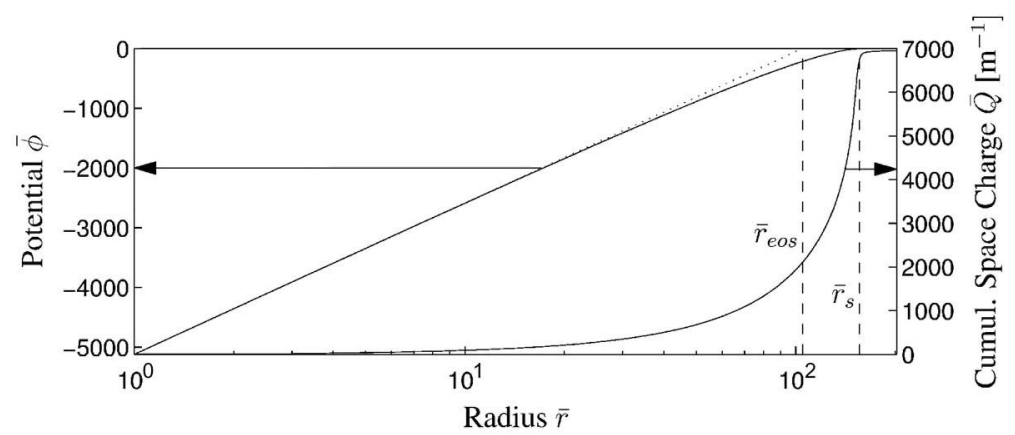

(a)

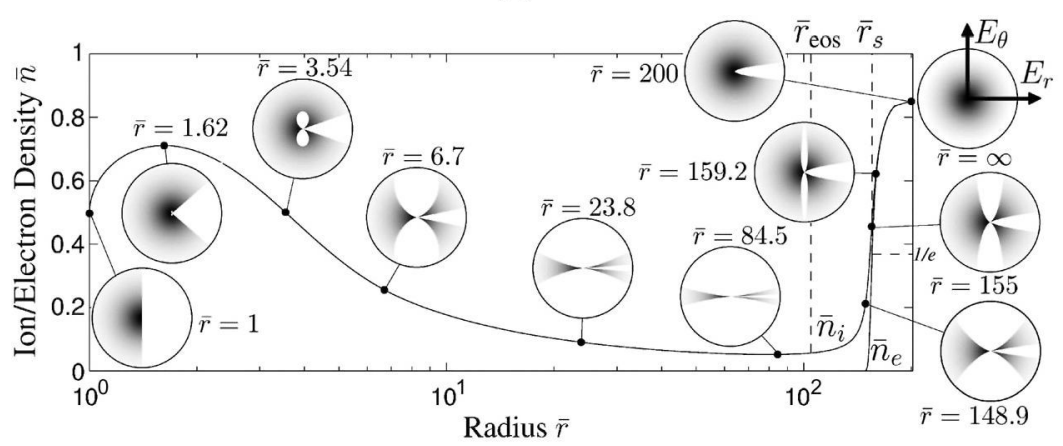

(b)

Fig. 2. Reproduction of a figure presented in [18, Figs. 3(b) and (c)]. The figure shows semilogarithmic profiles at values $T_{i}=T_{e} \equiv T, e \Phi_{p}=5120 \mathrm{kT}$, and $R=\lambda_{D}$ for (a) normalized potential $e \Phi / k T$ and cumulative space charge and (b) densities of attracted and repelled species (in addition to samples of the directional-energy distribution of the attracted species $E_{\theta} / E_{r}$ along the profile).

electric potential, electron density, and radius are normalized as in $[18]$

$$
\bar{\Phi} \equiv e \Phi / k T_{e}>0 \quad \bar{n} \equiv n_{e} / n_{\infty} \quad \bar{r} \equiv r / \lambda_{D}
$$

except that the relative potential $\Phi$ assumes positive values here. All dimensionless features in the profiles are functions of just three dimensionless parameters, which are the temperature, bias, and Debye ratios

$$
T_{i} / T_{e} \quad e \Phi_{p} / k T_{e} \equiv \bar{\Phi}_{p} \quad R / \lambda_{D} \equiv \bar{R}
$$

In this section, we compare theoretical results with numerical results from [18, Fig. 3(b) and (c)] [reproduced here, for ease of reference, as Fig. 2(a) and (b)], although no perfect comparison is possible with the available data. The parameter values

$$
T_{i} / T_{e}=1 \quad \bar{\Phi}_{p}=5120 \quad \bar{R}=1
$$

were used in Fig. 2, whereas $R$ was the maximum radius $R_{\max }$ collecting OML current throughout [6]. In [6, Fig. 6], one has

$$
\bar{R}_{\text {max }}\left(T_{i} / T_{e}=1, \bar{\Phi}_{p}=5120\right) \approx 1.09 .
$$

The semilogarithmic profiles shown in Fig. 2(a) numerically verify that the space charge has a negligible effect on the potential over a large neighborhood around the probe. The radius $r_{\text {eos }}$ in Fig. 2(a) corresponds to the zero-potential intercept in the symmetrical Laplace no-space-charge solution for cylindrical geometry

$$
\frac{\Phi}{\Phi_{p}}=\frac{\ln \left(r_{\mathrm{eos}} / r\right)}{\ln \left(r_{\mathrm{eos}} / R\right)} .
$$

The density profile of the attracted species shown in Fig. 2(b) exhibits the following five characteristic values: a "sheath" radius $r_{s}$, defined in [18] as corresponding to a potential $\Phi_{s}=$ $k T_{e} / e$; radii within the sheath $r\left(n_{\min }\right)$ and $r\left(n_{\max }\right)$, where the attracted-species density assumes minimum and maximum values, respectively; and the corresponding values $n_{\min }$ and $n_{\max }$. Numerical results for the aforementioned dimensionless characteristic values are

$$
\begin{aligned}
\bar{r}_{s} & =155.5 \\
\frac{\bar{r}_{s}}{\bar{r}_{\text {eos }}} & =\frac{155.5}{104.8} \approx 1.484 \\
\frac{\bar{r}\left(n_{\min }\right)}{\bar{r}_{\text {eos }}} & =\frac{80.2}{104.8} \approx 0.765 \\
\bar{n}_{\min } & =0.052 \\
\bar{r}\left(n_{\max }\right) & =1.62 \\
\bar{n}_{\max } & =0.71 .
\end{aligned}
$$

\section{A. Simple Sheath Features}

The high-bias asymptotic analysis in [6] introduces a thin layer between two close points, 1 and 2 in Fig. 1, where $d \Phi / d r$ in the quasi-neutral limit and $\Phi$ diverge, respectively. The plasma is quasi-neutral for $r>r_{1}$; no ions are present for $r<$ $r_{2}$. From [6, Appendix A, eq. (A4a) and following], one has

$$
\frac{r_{2}}{r_{1}}=1-3.45\left(\frac{2}{\lambda \mu}\right)^{1 / 5}\left(\sigma_{1} / \bar{\Phi}_{p} \bar{R}^{2}\right)^{2 / 5}
$$


where the product coefficient $\lambda \mu$, the coefficient

$$
\sigma_{1} \equiv \bar{\Phi}_{p} R^{2} / r_{1}^{2}
$$

and a third coefficient $\kappa$ used in the following to describe the density within the sheath only depend on the ratio $T_{i} / T_{e}$ in case $R=R_{\max }$. For $T_{i} / T_{e}=1$, one has (see [6, Fig. 5])

$$
\mu \lambda \approx 5.0 \quad \sigma_{1} \approx 0.24 \quad \kappa \approx 3.40
$$

here resulting in $r_{1} / r_{2} \approx 1.05$. With $\bar{\Phi}_{1}=O(1)<1$ and $\bar{\Phi}_{2}$ being large, our prediction for the sheath radius as defined in [18] $\left(\bar{\Phi}_{s}=1\right)$ would be $r_{s} \approx r_{1}$ (slightly smaller than $r_{1}$ ) or

$$
\begin{aligned}
\bar{r}_{s} & \approx \frac{R_{\max }}{\lambda_{D}} \frac{r_{1}}{R_{\max }} \\
& \approx \bar{R}_{\max } \times \sqrt{\frac{\bar{\Phi}_{p}}{\sigma_{1}}} \\
& \approx 1.09 \times \sqrt{\frac{5120}{0.24}} \\
& \approx 159.2
\end{aligned}
$$

as compared with 155.5 in (2a).

For the high-bias large-sheath cases considered here, the potential could always be written as [6], [7]

$$
\Phi \propto g(u) \quad u \equiv \ln \left(r_{2} / r\right)
$$

where the function $g$ was numerically determined. At a large $u$ (near the probe), $g$ takes a form corresponding to a no-spacecharge Laplace solution

$$
g(u) \approx c(u-b) \quad c \approx 2.09 \quad b \approx 0.351
$$

resulting in

$$
\frac{\Phi}{\Phi_{p}} \approx \frac{\ln \left(r_{2} / r\right)-b}{\ln \left(r_{2} / R\right)-b} \Rightarrow r_{\mathrm{eos}}=r_{2} e^{-b}
$$

leading to

$$
\frac{r_{s}}{r_{\mathrm{eos}}} \approx \frac{r_{1}}{r_{2}} \exp (b)=1.05 e^{0.351} \approx 1.490
$$

which compares with the ratio 1.484 in (2b).

Except near the probe (see in the following), the density within the sheath, as given in [6, eq. (20)], behaves as

$$
\bar{n}=\frac{\kappa}{\pi} \frac{R}{r} \sqrt{\frac{\Phi_{p}}{\Phi}} \approx \frac{\kappa}{\pi} \frac{R}{r_{2}} \sqrt{e^{2 u} \frac{c \ln \left(e^{-b} r_{2} / R\right)}{g(u)}} .
$$

The expression above has a minimum at point $m$ in Fig. 1, away from both probe and sheath boundaries, where $\Phi r^{2}$ goes through a maximum, i.e., where $g(u) e^{-2 u}$ is maximum. It follows from the numerical solution for $g(u)$ that $u_{m} \approx 0.63$ and $g\left(u_{m}\right) \approx 0.86[6]$. We then find that

$$
r\left(n_{\min }\right)=r_{2} e^{-0.63} \Rightarrow r\left(n_{\min }\right) / r_{\text {eos }}=e^{0.351-0.63} \approx 0.756
$$

as compared with a ratio of 0.765 in (2c).
Setting $u=0.63$ and $g \approx 0.86$ in (10) and using

$$
\frac{R}{r_{2}}=\frac{r_{1}}{r_{2}} \sqrt{\frac{\sigma_{1}}{\bar{\Phi}_{p}}}=1.05 \sqrt{\frac{0.24}{5120}} \approx 0.00719
$$

we find a density minimum

$$
\bar{n}_{\min } \approx 0.049
$$

to be compared with the value 0.052 in (2d).

\section{B. Density Maximum}

The sheath law $\bar{n} \propto 1 /(r \sqrt{ } \Phi)$, as given by (10), exhibits no maximum. As noted in [6, eq. (25)], however, that law fails near the probe, although this failure does not affect the solution for the potential, any space charge in a limited neighborhood around the probe having negligible effects because of the high bias. The maximum, which was noticed in 2001 [22] and again in 2003 [14], is explained in the following in terms of both the probe (as charge sink) and potential barrier effects. We recall here that in the absence of any such effect, the density of attracted particles in an arbitrary 2-D potential field remains undisturbed $\bar{n}=1$ throughout [20].

To allow for a possible density maximum, we then revert to a general expression in [6, eq. (7)]

$$
\bar{n}=\int_{0}^{\infty} \frac{d E}{\pi k T_{e}} \exp \left(\frac{-E}{k T_{e}}\right)\left[2 \sin ^{-1} \frac{J_{r}^{*}(E)}{J_{r}(E)}-\sin ^{-1} \frac{J_{R}^{*}(E)}{J_{r}(E)}\right]
$$

with

$$
\begin{aligned}
& J_{r}^{2}(E) \equiv 2 m_{e} r^{2}[E+e \Phi(r)] \\
& J_{r}^{*}(E) \equiv \min \left\lfloor J_{r^{\prime}}(E) ; r<r^{\prime}<\infty\right\rfloor \leq J_{r}(E) .
\end{aligned}
$$

For given $r$ and (electron energy) $E$, angular momentum values of zero and $J_{r}(E)$ correspond to zero azimuthal and radial velocity, respectively. In case $J_{r}^{*}(E)<J_{r}(E)$, inward trajectories with angular momentum in the range $J_{r}^{*}(E)<$ angular momentum $<J_{r}(E)$, which have low radial velocities, are unpopulated. Such trajectories, if traced back in time, turn around at radii between $r$ and the radius where the minimum in (16) occurs; a potential barrier is said to exist at $r$ [7].

We clearly have $J_{r}^{*}(E) \equiv J_{r}(E)$ whenever $J_{r}^{*}(0)=J_{r}(0)$ applies, i.e., for $r^{2} \Phi(r)<r^{\prime 2} \Phi\left(r^{\prime}\right)$ holding throughout the range, $r<r^{\prime}<\infty$. In Fig. 1, this applies for both $r>r_{0}$ and $r_{Q}>r>R$. The OML current law itself holds if there is no potential barrier at just $R$, i.e., there are no trajectories that, if traced back in time, turn around and return to the probe; the OML condition $R^{2} \Phi_{p}<r^{2} \Phi(r)$ for all $r$ requires point 0 to lie above the diagonal, as shown in Fig. 1. The second term in the bracket of (14) is the probe-as-sink effect. With $J_{R}^{*} \leq J_{R} \equiv \sqrt{2 m_{e} R^{2}\left(E+e \Phi_{p}\right)}$, that term vanishes with the probe size. In case one had $R \rightarrow 0$ and $J_{r}^{*}(E) \equiv J_{r}(E)$, (1) would indeed yield $\bar{n}=1$ as required in [20]. 
With $J_{R}^{*}(E) \equiv J_{R}(E)$ and $J_{r}^{*}(E) \equiv J_{r}(E)$ holding from point $Q$ to the probe in Fig. 1, (14) can be rewritten as

$$
\begin{aligned}
1-\bar{n} & =\int_{0}^{\infty} \frac{d E / k T_{e}}{\pi} \exp \left(\frac{-E}{k T_{e}}\right) \sin ^{-1} \sqrt{\frac{R^{2} \Phi_{p}}{r^{2} \Phi}} \\
& =\frac{1}{\pi} \sin ^{-1} \sqrt{\frac{R^{2} \Phi_{p}}{r^{2} \Phi}}
\end{aligned}
$$

where we used the approximation $k T_{e} \sim E \ll e \Phi_{Q}<e \Phi_{p}$, leading to $J_{r}(E) \approx J_{r}(0)$ for both $r_{Q}$ and $R$. Note that (14) recovers (10) only away from both probe and sheath boundaries, when both angles in the bracket of (14) are small, with $J_{r} \approx J_{r}(0) \propto r \sqrt{ } \Phi$, and $J_{r}^{*}(E)$ approximately given in [6] in terms of both $J_{r 0}(E)$ and $J_{r 1}(E)$, which determined the factor $\kappa$.

Clearly, the density increases along with $r^{2} \Phi$ from the probe to point $Q$ in Fig. 1 . If the density maximum actually occurred at $Q$, we could make a comparison on density maximum values by consistency, by using (1) for the potential not far from the probe in (17)

$$
\bar{n}_{\max }=\bar{n}_{Q}=1-\frac{1}{\pi} \sin ^{-1}\left[\frac{\bar{R}}{\bar{r}_{Q}} \sqrt{\frac{\ln \left(\bar{r}_{\mathrm{eos}} / \bar{R}\right.}{\ln \left(\bar{r}_{\mathrm{eos}} / \bar{r}_{Q}\right)}}\right] .
$$

When setting $\bar{R}=1, \bar{r}_{\text {eos }}=104.8$ and $\bar{r}_{Q}=\bar{r}\left(\bar{n}_{\max }\right)=1.62$ from (2e), (18) yields

$$
\bar{n}_{\max } \approx 0.77
$$

whereas we should have $\bar{n}_{\max } \approx 0.71$, as in (2f).

Point $Q$ is actually not quite the point of maximum density in Fig. 1. As one moves on the profile outward from $Q$, the second term in the bracket of (14) keeps decreasing as $r^{2} \Phi$ keeps increasing. We will now have $J_{r}^{*}<J_{r}$, with the first term in the bracket decreasing below its original unit value as well; however, this decrease can be shown to be slower at the start. Thus, $Q$ lies at some radius $\bar{r}_{Q}<\bar{r}\left(\bar{n}_{\max }\right)=1.62$, reducing the density maximum in (18).

\section{COMParison of Parametric Laws}

\section{A. Density-Minimum Law for $R=R_{\max }$}

Using (4) in (10) provides a general expression for the density minimum

$$
\bar{n}_{\min }=\frac{\kappa}{\pi} \frac{r_{1}}{r_{2}} \sqrt{\frac{\sigma_{1}}{\bar{\Phi}_{p}}} \sqrt{c \frac{\exp \left(2 u_{m}\right)}{g\left(u_{m}\right)}} \sqrt{\frac{1}{2} \ln \frac{\bar{\Phi}_{p}}{\sigma_{1}}-\ln \frac{r_{1}}{r_{2}}-b}
$$

with the ratio $r_{2} / r_{1}$ given by (3). Considering $\bar{R}=$ $\bar{R}_{\max }\left(\bar{\Phi}_{p}, T_{i} / T_{e}\right)$ with the values for $\lambda \mu, \sigma_{1}$ and $\kappa$ previously given for $T_{i} / T_{e}=1$, we find

$$
\bar{n}_{\min }\left(\Phi_{p}\right)=1.097 \frac{r_{1}}{r_{2}} \sqrt{\frac{\ln \bar{\Phi}_{p}+0.725-2 \ln \left(r_{1} / r_{2}\right)}{\bar{\Phi}_{p}}}
$$

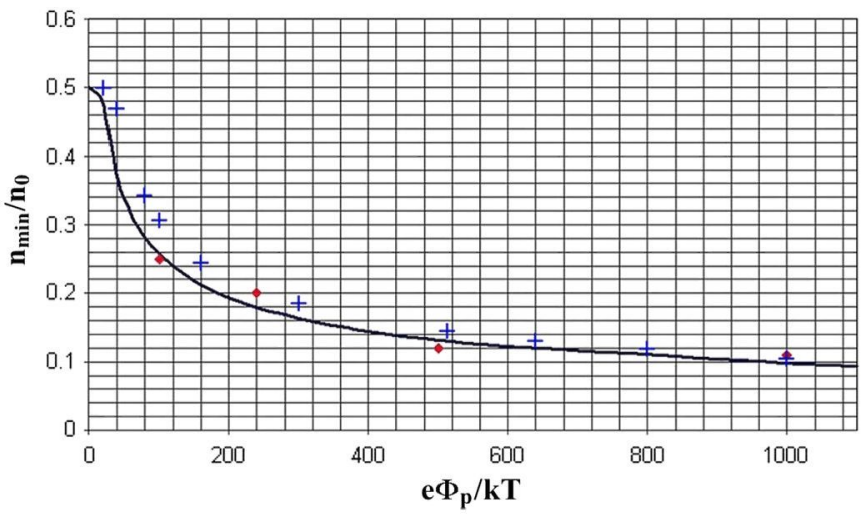

Fig. 3. Reproduction of a figure presented in [14, Fig. 5-3]. The figure compares results on a normalized density-minimum $n_{\min } / n_{\infty}$ versus probebias law from the theoretical law in (21a) and (21b) for (continuous line) $R=$ $R_{\max } \approx \lambda_{D}$ and (dots) PIC simulations for $R=\lambda_{D}$. It also incorporates results from (crosses) new KiPS-1-D simulations.

$$
\frac{r_{2}}{r_{1}}\left(\bar{\Phi}_{p}\right)=1-\frac{1.62}{\left[\sqrt{\Phi_{p}} \bar{R}_{\max }\left(\bar{\Phi}_{p}\right)\right]^{4 / 5}} .
$$

Fig. 3, which is a reproduction of a figure in [14, Fig. 5-3], compares density-minimum results from PIC simulations with the law in (21a) and (21b); the figure also incorporates new results from KiPS-1-D simulations. Note, however, that the simulations used both $T_{i} / T_{e}=1$ and $\bar{R}=1$ throughout, whereas $\bar{R}_{\max }$, as considered in (21a) and (21b), ranges from 0.7 to just above unity in [6, Fig. 6]. Moreover, the theoretical law was simplified in Fig. 3 by setting $r_{1} / r_{2}=1$, which is an approximation that is seen in (21b) to increasingly fail with the decreasing bias. The law itself should fail at a moderate/low bias. At zero bias, however, the minimum $\left(\bar{n}_{\min }=1 / 2\right)$ does occur at the probe, as shown in Fig. 3; for $\Phi_{p}=0$, one has $\Phi(r) \equiv 0$ and $n_{e}(r) \equiv n_{i}(r)$, which are particle densities having purely geometrical no-particle-mass dependence.

\section{B. Probe-Current Law for $R>R_{\max }$}

For $\bar{R}>\bar{R}_{\max }\left(\bar{\Phi}_{p}, T_{i} / T_{e}\right)$, the current to the probe falls below the OML value, which, at the high bias of interest, is $I_{\mathrm{OML}}=2 R L e n_{\infty} \sqrt{2 e \Phi_{p} / m_{e}}$. One then has

$$
\frac{I}{I_{\mathrm{OML}}}=G\left(\bar{\Phi}_{p}, \bar{R}, \frac{T_{i}}{T_{e}}\right) \quad \bar{R}>\bar{R}_{\max }\left(\bar{\Phi}_{p}, \frac{T_{i}}{T_{e}}\right) .
$$

The current ratio above was shown in [7] to decrease both with an increasing temperature ratio and (weakly) with a decreasing bias. Neglecting the weak dependence on the bias in $G$ and in $\bar{R}$ itself, $G$ could be written roughly in terms of a single variable [7]

$$
I / I_{\mathrm{OML}} \approx G\left[\bar{R}-\bar{R}_{\max }\left(T_{i} / T_{e}\right)\right] .
$$

Fig. 4, which is a reproduction of a figure found in [14, Fig. 5-15], compares values of $G$ from PIC simulation results with theoretical results from [7]. The simulations used $T_{i} / T_{e}=1$ and moderately low values of the bias. Note that differences are typically on the order of a few percent. 


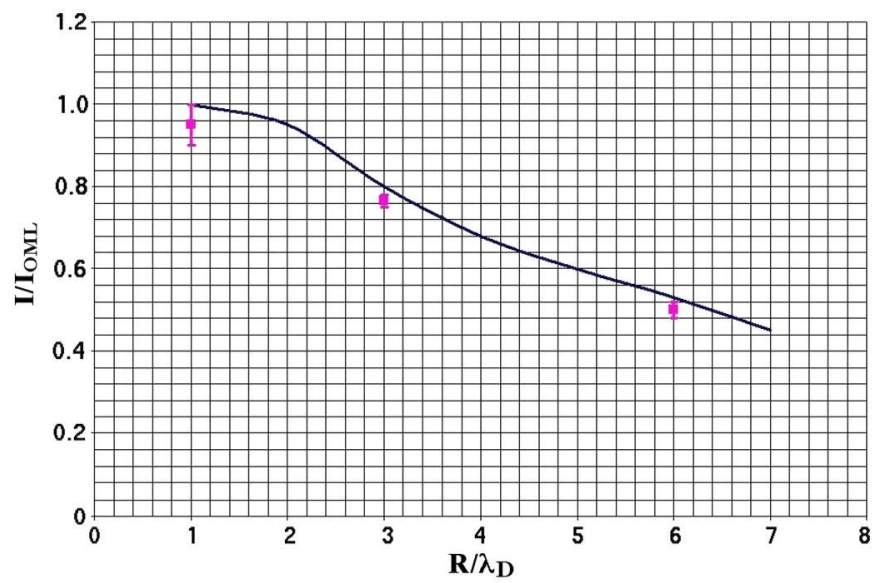

Fig. 4. Reproduction of a figure presented in [14, Fig. 5-15]. The figure compares results on the current drop below the OML value versus the probe radius for $R>R_{\max }$ from (continuous line) theoretical results in [7] and PIC simulations (showing error bars), with a weak dependence on bias ignored.

\section{Sheath Law for $R \ll R_{\max }$}

The case $R<R_{\max }$ was briefly mentioned in [7]. A sheathsize law can be derived, however, from [7, eq. (26)], which reads

$$
g\left[\ln \left(r_{2} / R\right)\right]=\left(\pi^{2} \sigma_{2} / \kappa^{2}\right)^{1 / 3} \bar{\Phi}_{p}^{1 / 3} / \bar{R}^{4 / 3}
$$

with $\sigma_{2} \equiv \sigma_{1} r_{1}^{2} / r_{2}^{2}, r_{2} / r_{1}$ as given in (3), and $\sigma_{1}$ as defined in (4). In general, both $\sigma_{1}$ and $\kappa$ are functions of all three ratios $T_{i} / T_{e}, \bar{\Phi}_{p}$, and $\bar{R}$. [For $R=R_{\max }=\lambda_{D} \bar{R}_{\max }\left(\bar{\Phi}_{p}, T_{i} / T_{e}\right)$, however, $\sigma_{1}$ and $\kappa$ are (as already noted) only functions of $T_{i} / T_{e}$, taking the respective values of 0.24 and 3.40 for the ratio $T_{i} / T_{e}=1$ considered here $]$.

The sheath law in the thin probe limit $\left(R \ll R_{\max } \sim \lambda_{D}\right)$ at a high bias is particularly simple because, as shown in the following, we then have

$$
\begin{aligned}
\sigma & \ll 1 \\
\kappa \sqrt{ } \sigma_{1} & =\text { constant } \equiv a=O(1) .
\end{aligned}
$$

Using the behavior in (7) for the function $g$, which is valid for large values of $\ln \left(r_{2} / R\right)$, and inserting (4) and (25b) in (24), we find

$$
\begin{aligned}
& c\left[\ln \left(r_{2} / R\right)-b\right] \approx\left(\pi^{2} \sigma_{1} \sigma_{2} / a^{2}\right)^{1 / 3} \bar{\Phi}_{p}^{1 / 3} / \bar{R}^{4 / 3} \\
\Rightarrow & c\left(\frac{a}{\pi}\right)^{2 / 3}\left(\frac{r_{1} r_{2}}{\lambda_{D}^{2}}\right)^{2 / 3}\left[\ln \left(\frac{r_{2}}{R}\right)-b\right]=\frac{e \Phi_{p}}{k T_{e}} .
\end{aligned}
$$

For $\bar{\Phi}_{p}$ large and both $a$ and $\bar{R}$ of order unity, (26a), with its left-hand side only logarithmically large, serves to verify that $\sigma_{1}$ is indeed small.

The numerically fitted sheath law for $R / \lambda_{D}=0.001$ presented in a figure in [18, Fig. 5] can be recovered from (26b) by neglecting $b$ against the logarithm of the very large ratio $r_{2} / R$ (leading to errors on the order of a few percent for the data used in [18]) and setting $r_{2} \approx r_{1} \approx r_{s}$

$$
c\left(\frac{a}{\pi}\right)^{2 / 3}\left(\frac{r_{s}}{\lambda_{D}}\right)^{4 / 3} \ln \left(\frac{r_{s}}{R}\right) \approx \frac{e \Phi_{p}}{k T_{e}}
$$

except that the exponent $4 / 3$ was there given as 1.346 . We will now calculate $a$ to compare $c(a / \pi)^{2 / 3}$ with the corresponding factor 1.298 in the numerical-fit law.

In what follows, we shall use the equations presented in [7, eqs. (18), (19), (22), (23), (25)], which involve energy $E$ integrals containing $J_{r}(E)$ functions for point 1 and for the probe $\left[J_{R}^{*}(E)=J_{R}(E)=J_{R}(0)\right]$, and a function $J_{\text {env }}(E)$, with $J^{2}=J_{\text {env }}^{2}(E)$ being some envelope of the family of straight lines $J^{2}=J_{r}^{2}(E)$ in the $E-J^{2}$ plane with $r$ as parameter [6], [21]. The second term inside a bracket in all those integrals can be neglected because the ratio

$$
\frac{J_{r 1}^{2}}{J_{R}^{2}}=\frac{r_{1}^{2}\left(E+e \Phi_{1}\right)}{R^{2} e \Phi_{p}}=\frac{\bar{\Phi}_{1}}{\sigma_{1}} \times\left(1+\frac{E}{e \Phi_{1}}\right)
$$

is large; $\bar{\Phi}_{1}$ is later found to be $O(1)$, and thus, $E \sim k T \sim e \Phi_{1}$.

The family envelope $J^{2}=J_{\text {env }}^{2}(E)$ arises from the appearance of potential barriers for $r<r_{0}$, as the profile shown in Fig. 1 goes through a minimum at point 0 and rapidly rises at the sheath entrance. The envelope was approximately given in terms of the $J^{2}=J_{r 0}^{2}(E), J^{2}=J_{r 1}^{2}(E)$ lines presented in an equation in [7, eq. (16)], which, using ansatzen $\rho \equiv r_{0}^{2} / r_{1}^{2}=$ $O(1), \Phi_{0} \ll \Phi_{1}$, reads

$$
\begin{aligned}
\frac{J_{\text {env }}^{2}}{J_{r 1}^{2}} & \approx 1-\frac{\bar{\Phi}_{1}^{2}}{\left(\bar{\Phi}_{1}+\varepsilon\right)\left[\bar{\Phi}_{1}+\varepsilon(\rho-1)\right]} \\
& =\frac{\rho w+(\rho-1) w^{2}}{1+\rho w+(\rho-1) w^{2}} \equiv \bar{J}^{2}(w, \rho) \\
w & \equiv \varepsilon / \bar{\Phi}_{1}=E / \Phi_{1} .
\end{aligned}
$$

The equation in [7, eq. (25)] now becomes

$$
\kappa \approx 2 \int_{0}^{\infty} d \varepsilon e^{-\varepsilon} \bar{J} \sqrt{1+\frac{\varepsilon}{\bar{\Phi}_{1}}} \sqrt{\frac{\bar{\Phi}_{1}}{\sigma_{1}}}
$$

which is just (25b), with $a$ given as

$$
a \equiv 2 \bar{\Phi}_{1}^{3 / 2} \int_{0}^{\infty} d w \exp \left(-\bar{\Phi}_{1} w\right) \bar{J}(w, \rho) \sqrt{1+w}
$$

To determine the values $\bar{\Phi}_{1}$ and $\rho$ as required to evaluate (31), one uses the aforementioned equations in [7, eqs. (18) and (19)], which read, respectively, as

$$
\begin{aligned}
& \exp \left(-\bar{\Phi}_{1}\right) \approx \frac{2}{\pi} \bar{\Phi}_{1} \int_{0}^{\infty} d w \exp \left(-\bar{\Phi}_{1} w\right) \sin ^{-1} \bar{J}(w, \rho) \\
& \exp \left(-\bar{\Phi}_{1}\right) \approx \frac{1}{\pi} \int_{0}^{\infty} \frac{d w \exp \left(-\bar{\Phi}_{1} w\right)}{1+w} \frac{\bar{J}(w, \rho)}{\sqrt{1-\bar{J}^{2}(w, \rho)}} .
\end{aligned}
$$

We find the values of $\bar{\Phi}_{1} \approx 0.350$ and $\rho \approx 1.724$, which are of order unity as advanced and are thus independent of $\bar{R}$ if small; when used in ( 31 ), we find that $a \approx 1.97$, finally yielding

$$
c(a / \pi)^{2 / 3} \approx 1.53
$$


Note that the value 1.298 in the numerical-fit law is about $15 \%$ smaller.

To understand this difference, which is greater than in the previous comparisons, note that both $\sigma_{1}$ and $\bar{R}^{2}$ are now small in (3). We then return to (26b), still neglecting $b$ and keeping $r_{s} \approx r_{1}$ but retaining (just outside the logarithmic term) the effect of a nonvanishing small difference $1-\left(r_{2} / r_{1}\right)$. From (3), using (4) to conveniently eliminate $\sigma_{1}$ and again using $r_{s} \approx r_{1}$, we find that

$$
\left(\frac{r_{2}}{r_{1}}\right)^{2 / 3} \approx 1-\frac{2}{3} \times 3.45\left(\frac{2}{\mu \lambda}\right)^{1 / 5}\left(\frac{\lambda_{D}}{r_{s}}\right)^{4 / 5}
$$

with the coefficients $\mu$ and $\lambda$ defined in general form in [7, eqs. (22) and (23)] (for $R=R_{\max }$, their product was given in [6], where $\lambda \mu \approx 5.0$, as noticed earlier). Those equations take simple forms for $R \ll R_{\max }$

$$
\begin{aligned}
\mu \approx \bar{\Phi}_{1} \int_{0}^{\infty} \frac{d w}{\pi} \exp \left(-\bar{\Phi}_{1} w\right) \frac{2 \bar{J}(w, \rho)}{\sqrt{1-\bar{J}^{2}(w, \rho)}} \\
\lambda=-\exp \left(-\bar{\Phi}_{1}\right)+\int_{0}^{\infty} \frac{d w}{2 \pi \bar{\Phi}_{1}} \exp \left(-\bar{\Phi}_{1} w\right) \\
\times \frac{\bar{J}(w, \rho)}{(1+w)^{2}} \frac{3-2 \bar{J}^{2}(w, \rho)}{\left[1-\bar{J}^{2}(w, \rho)\right]}
\end{aligned}
$$

yielding $\mu \approx 2.034$ and $\lambda \approx 0.5742$, respectively, when the previously found values of $\bar{\Phi}_{1} \approx 0.350$ and $\rho \approx 1.724$ are used. Equation (26b) then reads

$$
1.53\left[1-2.56\left(\frac{\lambda_{D}}{r_{s}}\right)^{4 / 5}\right]\left(\frac{r_{s}}{\lambda_{D}}\right)^{4 / 3} \ln \left(\frac{r_{s}}{R}\right) \approx \frac{e \Phi_{p}}{k T_{e}} .
$$

Equation (37) first shows that a simple law such as (27) somehow oversimplifies the true functional dependence of the sheath size of a very thin probe, whether one uses the numerical-fit factor 1.298 or the theoretical factor 1.53. Next, note that at a small given $R / \lambda_{D}$, the ratio $r_{s} / \lambda_{D}$ increases with the bias ratio $e \Phi_{p} / k T$ in (37), the value 1.53 thus being the correct limit factor in (27) at a very high bias, with the exponent $4 / 3$ as the right exponent. Moreover, note that the product 1.53 times the bracket in (37) is equal to 1.298 about midway through the $r_{s} / \lambda_{D}$ range used for the fitting in [18] (10-80, roughly). However, for a lower $r_{s} / \lambda_{D}$ and, thus, lower $e \Phi_{p} / k T$, the approximation $r_{s}=r_{1}$ breaks down, as does the agreement with the numerical results. Thus, (37) is only valid for the very high bias regime of interest for tether applications.

\section{CONClusion}

Analytical results on current collection by a cylindrical Langmuir probe at rest in unmagnetized plasma were compared with results from both steady-state Vlasov and PIC simulations. A probe bias that is very much greater than the plasma temperature (assumed equal for ions and electrons), as of interest for bare conductive tethers, was considered; results on probe current apply to a bare conductive tether because the bias along the tether varies over distances that are much larger than the local sheath size. At such a bias, both electric potential and attracted-species density exhibit complex radial profiles; in particular, the density exhibits a minimum that is well within the plasma sheath and a maximum that is closer to the probe. Excellent agreement was found between analytical and numerical results, for a probe radius $R$ near the maximum radius $R_{\max }$ for OML collection and a bias ratio near 5000, in the following profile features: value and position of density minimum; a relation between the value and position of the density maximum; the position of the sheath boundary; and the value of a radius characterizing the no-space-charge behavior of the potential near the high-bias probe.

Good agreement between the theory and simulations was also found for parametric laws jointly covering the following three characteristic $R$ ranges: sheath radius versus probe radius and bias, for $R \ll R_{\max }$; density minimum versus probe bias, for $R \cong R_{\max }$; and (weakly bias-dependent) current drop below the OML value versus probe radius, for $R>R_{\max }$.

The asymptotic analysis requires a large bias ratio $e \Phi_{p} / k T_{e}$, yielding a sheath-to-probe size ratio on the order of $1 / \sqrt{k T_{e} / e \Phi_{p}}$ for $R / R_{\max }$ or $R / \lambda_{D}$ of order unity. This makes for errors in the theory of $1 \%-3 \%$ for the bias of interest in tether applications. Results in [6] and [7] were also given for values of the temperature ratio $T_{i} / T_{e} \neq 1$; a comparison with corresponding numerical results should show an agreement similar to that found here as long as that ratio is of order unity. More important for future work is any possible dependence on a relative plasma velocity $U_{0}$. A fundamental difference between the negative and positive biases for the LEO $U_{0} \neq 0$ case, due to the mesothermal character of the relative plasma flow, might involve trapped-electron effects [15], [19].

\section{REFERENCES}

[1] J. R. Sanmartín, M. Martínez-Sánchez, and E. Ahedo, "Bare wire anodes for electrodynamic tethers," J. Propuls. Power, vol. 9, no. 3, pp. 353-360, May/Jun. 1993

[2] P. M. Chung, L. Talbot, and K. J. Touryan, Electric Probes in Flowing and Stationary Plasmas: Theory and Applications. New York: SpringerVerlag, 1975.

[3] J. C. McMahon, G. Z. Xu, and J. G. Laframboise, "The effect of ion drift on the sheath, presheath, and ion-current collection for cylinders in a collisionless plasma," Phys. Plasmas, vol. 12, no. 6, pp. 062 109-1062 109-11, Jun. 2005.

[4] S. D. Hester and A. A. Sonin, "Ion temperature sensitive end effect in cylindrical probe response at ionospheric satellite conditions," Phys. Fluids, vol. 13, no. 5, pp. 1265-1274, May 1970.

[5] J. R. Sanmartín, "End effect in Langmuir probe response under ionospheric satellite conditions," Phys. Fluids, vol. 15, no. 6, pp. 11341143, Jun. 1972.

[6] J. R. Sanmartín and R. D. Estes, "The orbital-motion-limited regime of cylindrical Langmuir probes," Phys. Plasmas, vol. 6, no. 1, pp. 395-405, Jan. 1999.

[7] R. D. Estes and J. R. Sanmartín, "Cylindrical Langmuir probes beyond the orbital-motion-limited regime," Phys. Plasmas, vol. 7, no. 10, pp. 4320 4325 , Oct. 2000.

[8] J. R. Sanmartín and R. D. Estes, "Interference of parallel cylindrical Langmuir probes," Phys. Plasmas, vol. 8, no. 9, pp. 4234-4239, Sep. 2001.

[9] B. E. Gilchrist, S. G. Bilén, and A. D. Gallimore, "Current collection to long, thin, probes in dense high-speed flowing plasma," in Proc. Space Technol. Appl. Int. Forum, M. S. El-Genk, Ed., New York, Am. Int. Phys., 2001, pp. 494-501. 
[10] B. E. Gilchrist, S. G. Bilén, and E. Choinière, "Analysis of chamber simulations of long collecting probes in high-speed dense plasmas," IEEE Trans. Plasma Sci., vol. 30, no. 5, pp. 2023-2034, Oct. 2002.

[11] E. Choinière, S. G. Bilén, B. E. Gilchrist, K. R. Fuhthop, and A. D. Gallimore, "Experimental investigation of electron collection to solid and slotted tape probes in a high-speed flowing plasma," IEEE Trans. Plasma Sci., vol. 33, no. 4, pp. 1310-1323, Aug. 2005.

[12] M. Kruijff, E. J. van der Heide, F. De Venuto, M. Dobrowolny, and G. Vannaroni, "Long term stability of bare conductive tethers: Combined results from plasma chamber tests and advanced simulations," presented at the Space Technology and Applications Int. Forum, Albuquerque, NM, Feb. 2001

[13] T. Onishi, "Numerical study of current collection by an orbiting bare tether," Ph.D. dissertation, MIT, Cambridge, MA, Aug. 2002.

[14] J.-B. Ferry, "Electron collection by an electrodynamic bare tether at high potential," M.S. thesis, MIT, Cambridge, MA, Jun. 2003.

[15] J.-M. Deux, "Kinetic modeling of electrodynamic space tethers," M.S. thesis, MIT, Cambridge, MA, Dec. 2004.

[16] E. Choinière and B. E. Gilchrist, "Kinetic modeling of the electron current collection to a moving bare electrodynamic tether," in Proc. Space Technol. Appl. Int. Fonum, M. S. El-Genk, Ed., New York, Am. Int. Phys., 2002, pp. 526-533.

[17] E. Choinière, "Theory and experimental evaluation of a consistent steadystate kinetic model for 2-D conductive structures in ionospheric plasmas with application to bare electrodynamic tethers in space," Ph.D. dissertation, Univ. Michigan, Ann Arbor, MI, Sep. 2004.

[18] E. Choinière and B. Gilchrist, "Selfconsistent 2-D kinetic simulations of high-voltage plasma sheaths surrounding ion-attracting conductive cylinders in flowing plasmas," IEEE Trans. Plasma Sci., vol. 35, no. 1, pp. 7-22, Feb. 2007.

[19] T. Onishi, M. Martínez-Sánchez, D. L. Cooke, and J. R. Sanmartín, "PIC computation of electron current collection to a moving bare tether in the mesothermal condition," presented at the 27th Int. Electric Propulsion Conf., Pasadena, CA, 2001, Paper IEPC-01-245. 